\title{
Construction of Astrophysical Initial Data for Perturbations of Relativistic Stars
}

\author{
Nils Andersson ${ }^{(1)}$, Kostas D. Kokkotas ${ }^{(2)}$, Pablo Laguna ${ }^{(3)}$, Philippos Papadopoulos ${ }^{(4)}$ and Michael S. Sipior ${ }^{(3)}$ \\ (1) Department of Mathematics \\ University of Southampton, Southampton SO17 1BJ, UK \\ (2) Department of Physics \\ Aristotle University of Thessaloniki \\ Thessaloniki 54006, Greece \\ (3) Department of Astronomy $\&$ Astrophysics and \\ Center for Gravitational Physics $\&$ Geometry \\ Penn State University, University Park, PA 16802, USA \\ (4) Max-Planck-Institut für Gravitationsphysik \\ Am Muhlenberg 5, 14476 Golm, Germany
}

\begin{abstract}
We develop a framework for constructing initial data sets for perturbations about spherically symmetric matter distributions. This framework facilitates setting initial data representing astrophysical sources of gravitational radiation involving relativistic stars. The procedure is based on Lichnerowicz-York's conformal approach to solve the constraints in Einstein's equations. The correspondence of these initial data sets in terms of the standard gauge perturbation variables in the Regge-Wheeler perturbation variables is established, and examples of initial data sets of merging neutron stars under the close-limit approximation are presented.
\end{abstract}

\section{INTRODUCTION}

The early years of the next millennium will hopefully be remembered for the birth of Gravitational-wave Astronomy. With several large scale interferometers (LIGO, VIRGO, GEO600, TAMA) under construction, and the continued improvement of the technology for cryogenic resonant-mass detectors (ALLEGRO, AURIGA, EXPLORER, NAUTILUS), there are many reasons to be optimistic at the present time. However, the interpretation of data from the new generation of detectors will heavily depend on accurate "templates" of gravitational wave-forms. For a given astrophysical source of gravitational radiation, construction of such templates involves fully non-linear or perturbative approximations to Einstein's field equations. In both instances, the construction of appropriate initial data constitutes a fundamental issue. It is absolutely necessary that initial data represent a "realistic" stage of the astrophysical system under consideration.

The early years of Numerical Relativity were in part characterized by studies aimed at constructing initial data for Einstein's equations, namely data that satisfies the Hamiltonian and momentum constraints. Of particular interest was obtaining solutions to the constraints which represent black hole binaries [1]. These initial data studies highlighted the importance that Lichnerowicz-York's conformal approach [2] plays in facilitating solving the constraints.

In the perturbative arena, initial data sets also play a fundamental role if a connection with systems of astrophysical relevance is to be made. Examples of perturbative studies where astrophysically consistent initial data is needed are the point-particle [3] and close-limit approximations [ $[$ ] to black hole coalescences. For the close-limit approximation in particular, this issue is crucial. The focus is then on the late stage of the merger, when the binary system can be approximated as a single, perturbed black hole. In the case of perturbations of relativistic stars, most of the studies [5] have not considered initial data with direct connection to a given astrophysical situation. In other words, the focus has so far been on investigating how the star reacts to generic perturbations.

The goal of this paper is to provide a mechanism for generating initial data for perturbations of a relativistic star. This data should ideally represent astrophysical situations of relevance to gravitational-wave detectors. Inspired by the success that Lichnerowicz-York's conformal approach has enjoyed for solving Einstein's constraints, we base our methodology on "linearizing Lichnerowicz-York's procedure". By doing so, we take advantage of the prescription for knowing which pieces of information, among the metric and its "velocity", are fixed by the constraints and which are freely specifiable. Moreover, we inherit the machinery used in the past for the construction of initial data sets representing binary systems. That is, the procedure described in this paper provides a natural framework for obtaining initial data in connection with the close-limit approximation to neutron star mergers $|6|$. As with black hole binaries, the close-limit to neutron star collisions deals with the late stages of the merger, at the point in which the systems can be approximated as a single neutron star "dressed" with perturbations. 


\section{LINEARIZATION OF LICHNEROWICZ-YORK'S CONFORMAL APPROACH}

Given a spacetime with 4-metric $\hat{g}_{\mu \nu}$ and a foliation of this spacetime with Eulerian observers having a 4-velocity $\hat{n}^{\mu}$, the constraints in Einstein's fields equations can be written in a 3+1 (or ADM) form [7] as

$$
\begin{aligned}
\hat{R}+\hat{K}^{2}-\hat{K}_{i j} \hat{K}^{i j} & =16 \pi \hat{\sigma} & & \text { (Hamiltonian) } \\
\hat{\nabla}_{j}\left(\hat{K}^{i j}-\hat{h}^{i j} \hat{K}\right) & =8 \pi \hat{j}^{i} . & & \text { (Momentum) }
\end{aligned}
$$

Above, $\hat{h}_{i j}$ is the 3 -metric $\left(\hat{h}_{\mu \nu}=\hat{g}_{\mu \nu}+\hat{n}_{\mu} \hat{n}_{\nu}\right)$ and $\hat{K}_{i j}$ the extrinsic curvature of the time-like hypersurfaces in the foliation, with $\hat{K}=\hat{h}^{i j} \hat{K}_{i j}$ and $\hat{R}$ the scalar curvature. Furthermore, $\hat{\nabla}_{i}$ is the covariant derivative associated with the 3-metric $\hat{h}_{i j}$, and $\hat{\sigma}$ and $\hat{j}^{i}$ are the energy and momentum densities of the matter sources. Greek letters denote spacetime indexes and Latin letters spatial indices, and we use units in which $G=c=1$. Contrary to the common practice, we use "hats" to denote physical space since we will be mostly working in the conformal space.

For a perfect fluid, the stress-energy tensor is given by

$$
\hat{T}_{\mu \nu}=(\hat{\rho}+\hat{p}) \hat{u}_{\mu} \hat{u}_{\nu}+\hat{p} \hat{g}_{\mu \nu}
$$

where $\hat{\rho}$ and $\hat{p}$ are respectively the total mass-energy density and pressure of the fluid measure by an observer with 4 -velocity $\hat{u}^{\mu}$. The energy and momentum densities appearing in the constraints are obtained from

$$
\begin{gathered}
\hat{\sigma}=\hat{T}_{\mu \nu} \hat{n}^{\mu} \hat{n}^{\nu}=(\hat{\rho}+\hat{p}) \hat{\gamma}^{2}-\hat{p} \\
\hat{j}^{\mu}=-\hat{T}_{\nu \alpha} \hat{n}^{\nu} \hat{h}^{\alpha \mu}=(\hat{\rho}+\hat{p}) \hat{\gamma} \hat{h}^{\mu \nu} \hat{u}_{\nu}
\end{gathered}
$$

where $\hat{\gamma} \equiv-\hat{n}_{\mu} \hat{u}^{\mu}$ is the relativistic boost factor.

The fundamental virtue of Lichnerowicz-York's conformal approach [2] for solving the Hamiltonian and momentum constraints is that it provides a concrete recipe for singling out which four "pieces" among the twelve components $\left(\hat{h}_{i j}, \hat{K}_{i j}\right)$ are to be solved from Eqs. (11) and (2). The starting point is the Ansatz

$$
\hat{h}_{i j}=\phi^{4} h_{i j},
$$

where the conformal metric $h_{i j}$ is assumed to be known. Thus, for the metric, the piece that is fixed by the constraints (Hamiltonian) is the conformal factor $\phi$. The other three quantities fixed by the constraints (momentum) involve the extrinsic curvature. The idea here is to decompose the extrinsic curvature into its trace, tracefree-transverse and tracefree-longitudinal parts. To achieve this, the extrinsic curvature is first split into

$$
\hat{K}^{i j}=\hat{A}^{i j}+\frac{1}{3} \hat{h}^{i j} \hat{K}
$$

Before decomposing the tracefree part $\hat{A}^{i j}$ into its transverse and longitudinal parts, the following conformal transformation is applied:

$$
\hat{A}^{i j}=\phi^{-10} A^{i j}
$$

The exponent in the conformal transformation is motivated by the fact that this transformation possesses the following property:

$$
\widehat{\nabla}_{j} \hat{A}^{i j}=\phi^{-10} \nabla_{j} A^{i j}
$$

with $\nabla_{i}$ covariant differentiation associated with the background metric $h_{i j}$. This property simplifies the conformal transformation of the divergence of the extrinsic curvature in the momentum constraint (2).

Since, as will become clear below, the trace of the extrinsic curvature $\hat{K}$ is not fixed by the constraints, no conformal transformation is imposed on the trace of the extrinsic curvature $(\hat{K}=K)$. Once the conformal transformation is applied, the next step is to decompose $A_{i j}$ into its transverse and longitudinal parts, namely

$$
A^{i j}=A_{*}^{i j}+(l W)^{i j},
$$

where 


$$
\begin{aligned}
(l W)^{i j} & =2 \nabla^{(i} W^{j)}-\frac{2}{3} h^{i j} \nabla_{k} W^{k}, \\
\nabla_{j} A_{*}^{i j} & =0 .
\end{aligned}
$$

With the above conformal transformations and transverse-longitudinal decompositions, the Hamiltonian and momentum constraints become

$$
\begin{aligned}
8 \nabla^{i} \nabla_{i} \phi-R \phi+A_{i j} A^{i j} \phi^{-7}-\frac{2}{3} K^{2} \phi^{5}+16 \pi \sigma \phi^{-3} & =0 \\
\nabla^{j} \nabla_{j} W^{i}+\frac{1}{3} \nabla^{i} \nabla_{j} W^{j}+R_{j}^{i} W^{j}-\frac{2}{3} \phi^{6} \nabla^{i} K-8 \pi j^{i} & =0,
\end{aligned}
$$

where $R^{i}{ }_{j}$ is the 3 -Ricci tensor of the conformal space and $R$ its trace. In deriving Eqs. (13) and (14), the following conformal transformations for the energy and momentum densities were used:

$$
\begin{gathered}
\hat{\sigma}=\phi^{-8} \sigma \\
\hat{j}^{i}=\phi^{-10} j^{i} .
\end{gathered}
$$

To summarize, the Hamiltonian constraint fixes the conformal factor $\phi$ and the momentum constraint determines the generator $W^{i}$ of the longitudinal part of the conformal, traceless part of the extrinsic curvature. The freely specifiable data in this coupled set of equations are the conformal metric $h_{i j}$, the trace of the extrinsic curvature $K$, the source functions $\left(\sigma, j^{i}\right)$, and the divergence free, traceless part of the extrinsic curvature $A_{*}^{i j}$, which is hidden in $A_{i j}$ in Eq. (13).

Thus far, we have just reviewed Lichnerowicz-York's treatment of the initial data problem. We now introduce our first assumption. The initial data, $\left(\hat{h}_{i j}, \hat{A}^{i j}, \hat{K}, \hat{\sigma}, \hat{j}^{i}\right)$, are assumed to be close to a given background, i.e.,

$$
\begin{aligned}
\hat{h}_{i j} & =\hat{h}_{i j}^{(0)}+\hat{h}_{i j}^{(1)} \\
\hat{A}^{i j} & =\hat{A}_{(0)}^{i j}+\hat{A}_{(1)}^{i j} \\
\hat{K} & =\hat{K}_{(0)}+\hat{K}_{(1)} \\
\hat{\sigma} & =\hat{\sigma}_{(0)}+\hat{\sigma}_{(1)} \\
\hat{j}^{i} & =\hat{j}_{(0)}^{i}+\hat{j}_{(1)}^{i},
\end{aligned}
$$

where ${ }^{(0)}$ labels background quantities and ${ }^{(1)}$ first-order perturbations. The corresponding decomposition of the conformal space quantities yields:

$$
\begin{aligned}
h_{i j} & =h_{i j}^{(0)}+h_{i j}^{(1)} \\
\phi & =\phi^{(0)}+\phi^{(1)} \\
A_{*}^{i j} & =A_{*(0)}^{i j}+A_{*(1)}^{i j} \\
W^{i} & =W_{(0)}^{i}+W_{(1)}^{i} \\
K & =K^{(0)}+K^{(1)} \\
\sigma & =\sigma^{(0)}+\sigma^{(1)} \\
j^{i} & =j_{(0)}^{i}+j_{(1)}^{i},
\end{aligned}
$$

where

$$
\begin{aligned}
& \hat{h}_{i j}^{(0)}=\phi_{(0)}^{4} h_{i j}^{(0)} \\
& \hat{h}_{i j}^{(1)}=\phi_{(0)}^{4} h_{i j}^{(1)}+4 \phi_{(0)}^{3} \phi_{(1)} h_{i j}^{(0)} \\
& \hat{A}_{(0)}^{i j}=\phi_{(0)}^{-10} A_{(0)}^{i j} \\
& \hat{A}_{(1)}^{i j}=\phi_{(0)}^{-10} A_{(1)}^{i j}-10 \phi_{(0)}^{-11} \phi_{(1)} A_{(0)}^{i j} \\
& \hat{K}_{(0)}=K_{(0)} \\
& \hat{K}_{(1)}=K_{(1)}
\end{aligned}
$$




$$
\begin{aligned}
& \hat{\sigma}_{(0)}=\phi_{(0)}^{-8} \sigma_{(0)} \\
& \hat{\sigma}_{(1)}=\phi_{(0)}^{-8} \sigma_{(1)}-8 \phi_{(0)}^{-9} \phi_{(1)} \sigma_{(0)} \\
& \hat{j}_{(0)}^{i}=\phi_{(0)}^{-10} j_{(0)}^{i} \\
& \hat{j}_{(1)}^{i}=\phi_{(0)}^{-10} j_{(1)}^{i}-10 \phi_{(0)}^{-11} \phi_{(1)} j_{(0)}^{i} .
\end{aligned}
$$

Although all scalars, vectors and tensors are expanded in order of smallness, it is important to stress again that all but $\phi$ and $W^{i}$ are freely specifiable, and this property is independent of the order of the perturbation.

At this point we introduce our second assumption, which is that the perturbations of the conformal background vanish on the initial data slice; that is, $h_{i j}^{(1)}=0$. The primary motivation for this choice is the simplification of the coupled system of constraint equations. We shall later discuss the physical relevance, as well as the implications and restrictions, that this assumption imposes on the class of initial data that one has access to with our procedure. Using $h_{i j}^{(1)}=0$, together with the above perturbative expansions in the coupled elliptic system (13) and (14), we obtain

$$
\begin{aligned}
& 8 \nabla^{j} \nabla_{j} \phi_{(1)}-[R+\left.7 A_{(0)}^{i j} A_{i j}^{(0)} \phi_{(0)}^{-8}+\frac{10}{3} K_{(0)}^{2} \phi_{(0)}^{4}+48 \pi \sigma_{(0)} \phi_{(0)}^{-4}\right] \phi_{(1)} \\
&+2 A_{i j}^{(0)} A_{(1)}^{i j} \phi_{(0)}^{-7}-\frac{4}{3} K_{(0)} K_{(1)} \phi_{(0)}^{5}-16 \pi \sigma_{(1)} \phi_{(0)}^{-3}=0 \\
& \nabla^{j} \nabla_{j} W_{(1)}^{i}+\frac{1}{3} \nabla^{i} \nabla_{j} W_{(1)}^{j}+R_{j}^{i} W_{(1)}^{j}-\frac{2}{3} \phi_{(0)}^{6} \nabla^{i} K_{(1)}-\frac{12}{3} \phi_{(0)}^{5} \phi_{(1)} \nabla^{i} K_{(0)}-8 \pi j_{(1)}^{i}=0
\end{aligned}
$$

where $R, R_{i j}$ and $\nabla_{i}$ refer to the background. In writing Eqs. (39) and (40), we have used the fact that the zeroth-order quantities satisfy the constraints. Notice that, as in the non-linear case, the constraints remain coupled.

\section{THE INITIAL DATA PROBLEM FOR RELATIVISTIC STELLAR PERTURBATIONS}

We now focus on constructing initial data sets for which the background is a static and spherically symmetric stellar model, with 4-metric given by

$$
d s^{2}=-e^{2 \nu} d t^{2}+e^{2 \lambda} d \hat{r}^{2}+\hat{r}^{2} d \theta^{2}+\hat{r}^{2} \sin ^{2} \theta d \varphi^{2}
$$

where the metric coefficients $\nu$ and $\lambda$ are functions of the radial coordinate $\hat{r}$ only. Einstein's equations for this background reduce to solving three equations. The first equation defines the "mass inside radius $\hat{r}$;

$$
\frac{d m}{d \hat{r}}=4 \pi \hat{r}^{2} \hat{\rho}_{(0)} .
$$

Here $m$ is a function of $\hat{r}$ that is related to the metric function $\lambda$ by

$$
e^{-2 \lambda} \equiv 1-\frac{2 m}{\hat{r}}
$$

Equation (42) is directly obtained from the Hamiltonian constraint. The second equation belongs to Einstein's evolution equations and reads

$$
\frac{d \nu}{d \hat{r}}=\frac{e^{2 \lambda}}{\hat{r}^{2}}\left(m+4 \pi \hat{r}^{3} \hat{p}_{(0)}\right) .
$$

Finally, conservation of momentum yields the condition for hydrostatic equilibrium:

$$
\frac{d \hat{p}_{(0)}}{d \hat{r}}=-\left(\hat{\rho}_{(0)}+\hat{p}_{(0)}\right) \frac{d \nu}{d \hat{r}}
$$

The above stellar structure system of equations (the Tolman-Oppenheimer-Volkoff equations) must be supplemented with an equation of state. For simplicity, we use the polytropic equation of state $\hat{p}=\kappa \hat{\rho}^{\Gamma}$, where $\kappa$ and $\Gamma$ are the adiabatic constant and index, respectively. The adiabatic index $\Gamma$ is related to the polytropic index $n$ by $\Gamma=1+1 / n$. In the specific example provided later, we use $\Gamma=2(n=1)$. 
We now assume that the zero-order quantities $\left(\hat{\rho}_{(0)}, \hat{p}_{(0)}, \lambda, \nu\right)$ have been obtained from solving Eqs. (42), 44) and (45). The next step is to solve for the first-order perturbations from the linearized constraints (39) and (40). To facilitate this task, it is convenient to perform a coordinate transformation and bring the 3-metric in (41) into the isotropic, conformally flat form:

$$
d s^{2}=\phi_{(0)}^{4}\left(d r^{2}+r^{2} d \theta^{2}+r^{2} \sin ^{2} \theta d \varphi^{2}\right),
$$

where the conformal factor is given by

$$
\phi_{(0)}=\left(\frac{\hat{r}}{r}\right)^{1 / 2}
$$

and the transformation of the radial-coordinate is obtained from

$$
\frac{d r}{d \hat{r}}=e^{\lambda} \frac{r}{\hat{r}} \text {. }
$$

The static nature of the background spacetime and the gauge choice of a vanishing shift vector imply $K_{i j}^{(0)}=j_{(0)}^{i}=0$ for this background spacetime. This leads to considerable simplifications in the following, but the procedure we describe for constructing perturbative initial data can easily be extended to include also time-dependent, spherically symmetric background spacetimes.

We introduce here our third assumption, which is that $A_{*(1)}^{i j}=K_{(1)}=0$. The vanishing of the transverse-traceless part of the perturbation to the extrinsic curvature has direct implications to the gravitational radiation content of the initial data. In a way this assumption can be viewed as "minimizing" the amount of gravitational waves in the initial spacetime. That this may not be desirable is obvious, but in order to be able to assign this part of the free data physically correct values we need a detailed knowledge of the past history of the system. Such information requires long term, nonlinear evolutions and is far beyond our present capabilities. The present assumption is convenient in that it simplifies the calculations considerably. Furthermore, we are unlikely to overestimate the amount of gravitational radiation emerging from true physical systems if we base our estimates on the present approach. The vanishing of the trace of the extrinsic curvature to first order is less restrictive physically. It simply implies (using also the fact that $\left.K_{(0)}=0\right)$ that the slicing of the perturbed spacetime is maximal to first order. With those further assumptions, the linearized constraints (39) and (40) decouple and take the form

$$
\begin{aligned}
\nabla^{j} \nabla_{j} \phi_{(1)} & =6 \pi \sigma_{(0)} \phi_{(0)}^{-4} \phi_{(1)}-2 \pi \phi_{(0)}^{-3} \sigma_{(1)} \\
\nabla^{i} \nabla_{i} U & =\nabla_{i} V^{i} \\
\nabla^{j} \nabla_{j} V^{i} & =8 \pi j_{(1)}^{i} .
\end{aligned}
$$

where we have decomposed the vector $W_{(1)}^{i}$ in Eq. (40) following [8,9] as

$$
W_{(1)}^{i}=V^{i}-\frac{1}{4} \nabla^{i} U
$$

From now on, we will drop the label ${ }_{(1)}$ in $j_{(1)}^{i}$ and $W_{(1)}^{i}$ since the zero-order values for these quantities vanish.

The set of equations 49-51 constitute a coupled set of elliptic equations in three dimensions, expressed e.g., in the coordinates $(r, \theta, \varphi)$ of the background space. Due to the spherically symmetric nature of the background, these linearized equations allow for a separation of variables. Specifically, we can apply a spherical harmonic decomposition of form:

$$
\begin{aligned}
\phi_{(1)}(r, \theta, \varphi) & =\sum_{l m} \phi_{(1)}(r) Y_{l m}(\theta, \varphi) \\
\sigma_{(1)}(r, \theta, \varphi) & =\sum_{l m} \sigma_{(1)}(r) Y_{l m}(\theta, \varphi) \\
U(r, \theta, \varphi) & =\sum_{l m} U(r) Y_{l m}(\theta, \varphi) \\
V^{i}(r, \theta, \varphi) & =\sum_{l m} V_{1}(r) e_{1}^{i}(\theta, \varphi)+r V_{2}(r) e_{2}^{i}(\theta, \varphi)+r V_{3}(r) e_{3}^{i}(\theta, \varphi) \\
W^{i}(r, \theta, \varphi) & =\sum_{l m} W_{1}(r) e_{1}^{i}(\theta, \varphi)+r W_{2}(r) e_{2}^{i}(\theta, \varphi)+r W_{3}(r) e_{3}^{i}(\theta, \varphi) \\
j^{i}(r, \theta, \varphi) & =\sum_{l m} J_{1}(r) e_{1}^{i}(\theta, \varphi)+r J_{2}(r) e_{2}^{i}(\theta, \varphi),+r J_{3}(r) e_{3}^{i}(\theta, \varphi),
\end{aligned}
$$


where

$$
\begin{aligned}
e_{1}^{i} & =\left(Y_{l m}, 0,0\right) \\
e_{2}^{i} & =\left(0, \frac{1}{r^{2}} \partial_{\theta} Y_{l m}, \frac{1}{r^{2} \sin ^{2} \theta} \partial_{\varphi} Y_{l m}\right) \\
e_{3}^{i} & =\left(0,-\frac{1}{r^{2} \sin \theta} \partial_{\varphi} Y_{l m}, \frac{1}{r^{2} \sin \theta} \partial_{\theta} Y_{l m}\right) .
\end{aligned}
$$

Here $e_{1}^{i}$ and $e_{2}^{i}$ are the basis vectors of even-parity perturbations and $e_{3}^{i}$ is the basis for odd-parity perturbations. In the above expressions and what follows, it is understood that the radial functions are for a given $(l, m)$. These indices have been suppressed for economy in notation. We note that in terms of the radial functions, Eq. (52) reduces to

$$
\begin{aligned}
& W_{1}=V_{1}-\frac{1}{4} \frac{d}{d r} U \\
& W_{2}=V_{2}-\frac{1}{4} U \\
& W_{3}=V_{3} .
\end{aligned}
$$

After separation of variables, the system of equations (49-51) is rewritten as a system of coupled radial elliptic equations:

$$
\begin{aligned}
\frac{1}{r^{2}} \frac{d}{d r}\left(r^{2} \frac{d}{d r} \phi_{(1)}\right)-\left[\frac{l(l+1)}{r^{2}}+6 \pi \sigma_{(0)} \phi_{(0)}^{-4}\right] \phi_{(1)} & =-2 \pi \phi_{(0)}^{-3} \sigma_{(1)} \\
\frac{1}{r^{2}} \frac{d}{d r}\left(r^{2} \frac{d}{d r} U\right)-\frac{l(l+1)}{r^{2}} U & =\frac{1}{r^{2}} \frac{d}{d r}\left(r^{2} V_{1}\right)-\frac{l(l+1)}{r} V_{2} \\
\frac{1}{r^{2}} \frac{d}{d r}\left(r^{2} \frac{d}{d r} V_{1}\right)-\frac{[l(l+1)+2]}{r^{2}} V_{1}+2 \frac{l(l+1)}{r^{2}} V_{2} & =8 \pi J_{1} \\
\frac{1}{r^{2}} \frac{d}{d r}\left(r^{2} \frac{d}{d r} V_{2}\right)-\frac{l(l+1)}{r^{2}} V_{2}+\frac{2}{r^{2}} V_{1} & =8 \pi J_{2} \\
\frac{1}{r^{2}} \frac{d}{d r}\left(r^{2} \frac{d}{d r} V_{3}\right)-\frac{l(l+1)}{r^{2}} V_{3} & =8 \pi J_{3} .
\end{aligned}
$$

Equations (61-65) fully characterize, for each $(l, m)$ harmonic, initial data $\left(\phi_{(1)}, U, V_{i}\right)$ to first perturbative order, once the background conformal factor $\phi_{(0)}$ and density $\sigma_{(0)}$ as well as the fluid perturbations $\sigma_{(1)}$ and $J_{i}$ are specified. Outside the sources, the solutions to the above equations are $U=u r^{a}, V_{1}=v_{1} r^{b}, V_{2}=v_{2} r^{c}$ and $V_{3}=v_{3} r^{d}$ with $u$, $v_{1}, v_{2}$ and $v_{3}$ constants. In order to have regular solutions for $r \rightarrow \infty$, one needs $a=d=-(l+1), b=c=-l$ and $v_{1}=l v_{2}$, where we assume that $l \geq 1$. As we shall later see, the corresponding interior solutions for head-on and inspiral close-limit collisions exhibit the same scaling; that is, $V_{1}=l V_{2}$ as well as $J_{1}=l J_{2}$. With this assumption, the system of equations 6265 reduces to

$$
\begin{aligned}
\frac{1}{r^{2}} \frac{d}{d r}\left(r^{2} \frac{d}{d r} U\right)-\frac{l(l+1)}{r^{2}} U & =\frac{d}{d r} V_{1}-\frac{(l-1)}{r} V_{1} \\
\frac{1}{r^{2}} \frac{d}{d r}\left(r^{2} \frac{d}{d r} V_{1}\right)-\frac{l(l-1)}{r^{2}} V_{1} & =8 \pi J_{1} \\
\frac{1}{r^{2}} \frac{d}{d r}\left(r^{2} \frac{d}{d r} V_{3}\right)-\frac{l(l+1)}{r^{2}} V_{3} & =8 \pi J_{3} .
\end{aligned}
$$

\section{CORRESPONDENCE WITH REGGE-WHEELER VARIABLES}

Before we proceed to present examples of initial data sets constructed using the above approach, we want to establish the correspondence between our variables and the standard Regge-Wheeler variables. This is relevant since the Regge-Wheeler notation (and the associated gauge) is customarily used in perturbative evolutions for spherical stellar models [5]. To this end, consider a spatial tensor $T_{i j}$ such that $T_{i j}(\hat{r}, \theta, \varphi)=T_{i j}^{(0)}(\hat{r})+T_{i j}^{(1)}(\hat{r}, \theta, \varphi)$. The perturbations of this tensor can be decomposed as 


$$
T_{i j}^{(1)}=t_{1}(\hat{r}) f_{i j}^{1}+\hat{r} t_{2}(\hat{r}) f_{i j}^{2}+\hat{r}^{2} t_{3}(\hat{r}) f_{i j}^{3}+\hat{r}^{2} t_{4}(\hat{r}) f_{i j}^{4}+t_{5}(\hat{r}) f_{i j}^{5}+\hat{t}_{6}(\hat{r}) f_{i j}^{6},
$$

where

$$
\begin{aligned}
& f_{i j}^{1}=\left(\begin{array}{ccc}
Y_{l m} & 0 & 0 \\
0 & 0 & 0 \\
0 & 0 & 0
\end{array}\right), \\
& f_{i j}^{2}=\left(\begin{array}{ccc}
0 & \partial_{\theta} Y_{l m} & \partial_{\varphi} Y_{l m} \\
\operatorname{symm} & 0 & 0 \\
\operatorname{symm} & 0 & 0
\end{array}\right) \\
& f_{i j}^{3}=\left(\begin{array}{ccc}
0 & 0 & 0 \\
0 & Y_{l m} & 0 \\
0 & 0 & \sin ^{2} \theta Y_{l m}
\end{array}\right) \\
& f_{i j}^{4}=\left(\begin{array}{ccc}
0 & 0 & 0 \\
0 & \partial_{\theta}^{2} Y_{l m} & \left(\partial_{\theta} \partial_{\varphi}-\cot \theta \partial_{\varphi}\right) Y_{l m} \\
0 & \operatorname{symm} & \left(\partial_{\varphi}^{2}+\sin \theta \cos \theta \partial_{\theta}\right) Y_{l m}
\end{array}\right) \\
& f_{i j}^{5}=\left(\begin{array}{ccc}
0 & -\frac{1}{\sin \theta} \partial_{\varphi} Y_{l m} & \sin \theta \partial_{\theta} Y_{l m} \\
\operatorname{symm} & 0 & 0 \\
\operatorname{symm} & 0 & 0
\end{array}\right), \\
& f_{i j}^{6}=\left(\begin{array}{ccc}
0 & 0 & 0 \\
0 & \frac{1}{\sin \theta}\left(\partial_{\theta} \partial_{\varphi}-\cot \theta\right) Y_{l m} & \frac{\sin \theta}{2}\left(\frac{1}{\sin ^{2} \theta} \partial_{\varphi}^{2}+\cot \theta \partial_{\theta}-\partial_{\theta}^{2}\right) Y_{l m} \\
0 & \operatorname{symm} & -\sin \theta\left(\partial_{\theta} \partial_{\varphi}-\cot \theta \partial_{\varphi}\right) Y_{l m}
\end{array}\right) .
\end{aligned}
$$

Above, $f_{i j}^{1}, f_{i j}^{2}, f_{i j}^{3}$, and $f_{i j}^{4}$ represent the even-parity tensor spherical harmonics and $f_{i j}^{5}$ and $f_{i j}^{6}$ the odd-parity counterparts.

Using the Regge-Wheeler notation, the perturbations of the spatial metric (in physical space) read

$$
\hat{h}_{i j}^{(1)}=\sum_{l m} e^{2 \lambda} H_{2}(\hat{r}) f_{i j}^{1}+h_{1}^{\text {even }}(\hat{r}) f_{i j}^{2}+\hat{r}^{2} K(\hat{r}) f_{i j}^{3}+\hat{r}^{2} G(\hat{r}) f_{i j}^{4}+h_{1}^{\text {odd }}(\hat{r}) f_{i j}^{5}+h_{2}(\hat{r}) f_{i j}^{6},
$$

where $K$ must not be confused with the trace of the extrinsic curvature. From the previous section, we have that the spatial metric can be constructed from

$$
\begin{aligned}
\hat{h}_{i j} & =\hat{h}_{i j}^{(0)}+\hat{h}_{i j}^{(1)}=\phi^{4} h_{i j}=\left(\phi_{(0)}+\phi_{(1)}\right)^{4} h_{i j}^{(0)} \\
& =\phi_{(0)}^{4} h_{i j}^{(0)}+4 \phi_{(0)}^{3} \phi_{(1)} h_{i j}^{(0)} \\
& =\hat{h}_{i j}^{(0)}+4 \frac{\phi_{(1)}}{\phi_{(0)}} \hat{h}_{i j}^{(0)},
\end{aligned}
$$

where $\phi_{(0)}=(\hat{r} / r)^{1 / 2}, \hat{h}_{i j}^{(0)}=\operatorname{diag}\left(e^{2 \lambda}, \hat{r}^{2}, \hat{r}^{2} \sin ^{2} \theta\right)$ and $\phi_{(1)}=\sum_{l m} \phi_{(1)}(r) Y_{l m}$ with $\phi_{(1)}(r)$ a solution of the radial equation (61). Thus, our approach to construct initial data yields spatial metric perturbations of the form

$$
\hat{h}_{i j}^{(1)}=\sum_{l m} 4 \phi_{(1)}(r)\left(\frac{\hat{r}}{r}\right)^{1 / 2}\left(e^{2 \lambda} f_{i j}^{1}+\hat{r}^{2} f_{i j}^{3}\right) .
$$

Comparison of the metric perturbations $(79)$ with $(76)$ shows that our procedure for constructing initial data yields in terms of the Regge-Wheeler notation $h_{1}^{\text {odd }}=h_{1}^{\text {even }}=h_{2}=G=0$ and

$$
H_{2}=K=4 \phi_{(1)}(r)\left(\frac{\hat{r}}{r}\right)^{1 / 2}
$$


Consider now the extrinsic curvature. As mentioned before, the extrinsic curvature vanishes to zero-order. In addition, we made the choice of having vanishing first-order trace $K_{(1)}$ and transverse-traceless parts $A_{*(1)}^{i j}$. Thus, the extrinsic curvature is completely determined by the vector $W^{i}$ and the conformal factor of the background space $\phi_{(0)}$ from

$$
\hat{K}_{i j}=\phi_{(0)}^{-2}(l W)_{i j}
$$

In terms of tensor spherical harmonics, the extrinsic curvature reads

$$
\hat{K}_{i j}=\sum_{l m}\left(\frac{r}{\hat{r}}\right)\left[k_{1}(r) f_{i j}^{1}+r k_{2}(r) f_{i j}^{2}+r^{2} k_{3}(r) f_{i j}^{3}+r^{2} k_{4}(r) f_{i j}^{4}+k_{5}(r) f_{i j}^{5}+k_{6}(r) f_{i j}^{6}\right],
$$

where

$$
\begin{aligned}
k_{1} & =\frac{2}{3 r}\left[2 r \frac{d}{d r} W_{1}+l(l+1) W_{2}-2 W_{1}\right] \\
k_{2} & =\frac{1}{r}\left[r \frac{d}{d r} W_{2}+W_{1}-W_{2}\right] \\
k_{3} & =\frac{2}{3 r}\left[-r \frac{d}{d r} W_{1}+W_{1}+l(l+1) W_{2}\right] \\
k_{4} & =\frac{2}{r} W_{2} \\
k_{5} & =r \frac{d}{d r} W_{3}-W_{3} \\
k_{6} & =-2 r W_{3} .
\end{aligned}
$$

Before proceeding, it is appropriate to discuss the implications of the assumptions we imposed for the presence of even and odd parity perturbations in the initial data.

Consider first the perturbations of the 3-metric: The assumption of vanishing perturbations of the conformal 3 -metric implies (as seen from Eq. (79)), that $h_{1}^{\text {odd }}=h_{1}^{\text {even }}=h_{2}=G=0$. This means that all odd parity perturbations of the 3-metric must vanish on the initial surface. This does not however exclude the presence of oddparity perturbations. Such perturbations may enter via the Regge-Wheeler variable $h_{0}$, which is freely specifiable. Similarly for even-parity perturbations, the Regge-Wheeler quantities $H_{0}$ and $H_{1}$ are not part of the constraints and can be chosen freely. A choice of these three variables correspond to choosing a slicing for the spacetime, i.e. specifying the lapse and the shift vector. Specifically, Regge-Wheeler gauge corresponds to $h_{1}^{\text {even }}=G=h_{2}=0$ above, as well as perturbed lapse

$$
\delta \alpha=e^{2 \nu} H_{0}
$$

and shift vector

$$
\delta \beta^{i}=H_{1} e_{1}^{i}+h_{0} e_{3}^{i} .
$$

Furthermore, there are no constraints on the odd or even parity nature of the extrinsic curvature initial data. This follows immediately from Eq. (82) since the coefficients $k_{1}$ through $k_{6}$ are in general non-vanishing. As a consequence, even if the initial 3-metric has vanishing odd-parity perturbations, their time evolution will in general include such perturbations.

\section{SAMPLE INITIAL DATA SETS: COLLIDING NEUTRON STARS}

We will now apply the method for constructing initial data, that was presented in Sec. III, to a case of astrophysical relevance. We consider collisions of neutron stars under the close-limit approximation. Related results have already been discussed by Allen et al [6]. That study was specialized to the case of head-on collisions. Here, we present a general discussion of neutron-star close-limit initial data, and give explicit results for both boosted head-on and inspiralling collisions. 
As stated at the end of Sec. III, initial data are obtained by solving the system of equations equations (61-65). The input for these equations are the background conformal factor $\phi_{(0)}$ and density $\sigma_{(0)}$ and, in addition, the fluid perturbations $\sigma_{(1)}$ and $J_{i}$. For close-limit collisions, one specifies the background from the outcome of the collisions. The fluid perturbations $\sigma_{(1)}$ and $J_{i}$, on the other hand, are obtained by "subtracting" the background from a suitable superposition of stars that represent the initial configuration. A suitable way of relating the two initial stars to the final configuration was presented in [6], and we refer the reader to that paper for further details.

Let us first consider the perturbation to the background density. Neglecting complicating factors, such as the effects from tidal deformations, we approximate the total density of the binary system with the following superposition of density profiles of isolated neutron stars $[6$ :

$$
\sigma\left(r^{i}\right)=\sigma_{*}\left(r^{i}-\xi^{i}\right)+\sigma_{*}\left(r^{i}+\xi^{i}\right)-\left[\sigma_{*}\left(r^{i}-\xi^{i}\right) \sigma_{*}\left(r^{i}+\xi^{i}\right)\right]^{1 / 2},
$$

where $\sigma_{*}$ is the conformally transformed density profile of the colliding neutron stars in isolation located a distant $\xi^{i}$ in conformal space. This functional form (87) for the total density is chosen since it leads to the correct zeroseparation and infinite-separation limits. That is, for zero-separation $\sigma \rightarrow \sigma_{*}$, and for large separations $\sigma\left(r^{i}\right) \rightarrow$ $\sigma_{*}\left(r^{i}-\xi^{i}\right)+\sigma_{*}\left(r^{i}+\xi^{i}\right)$. We now introduce the close-limit approximation, $\xi^{i} \ll 1$, and write

$$
\sigma_{*}\left(r^{i} \pm \xi^{i}\right)=\sigma_{*}\left(r^{i}\right) \pm \xi^{i} \nabla_{i} \sigma_{*}\left(r^{i}\right)+\frac{1}{2} \xi^{i} \xi^{j} \nabla_{i} \nabla_{j} \sigma_{*}\left(r^{i}\right) .
$$

Therefore, Eq. (87) takes the form

$$
\sigma=\sigma_{*}+\frac{1}{2}\left(\xi^{i} \nabla_{i} \sigma_{*}\right)^{2}+\frac{1}{2} \xi^{i} \xi^{j} \nabla_{i} \nabla_{j} \sigma_{*}
$$

Given this total energy, the density perturbation is obtained from

$$
\begin{aligned}
\sigma_{(1)} & =\sigma-\sigma_{(0)} \\
& =\sigma_{*}-\sigma_{(0)}+\frac{1}{2}\left(\xi^{i} \nabla_{i} \sigma_{*}\right)^{2}+\frac{1}{2} \xi^{i} \xi^{j} \nabla_{i} \nabla_{j} \sigma_{*} .
\end{aligned}
$$

Similarly, the momentum density is given by the superposition of momentum densities of boosted isolated stars:

$$
j^{i}\left(r^{k}\right)=j_{+}^{i}\left(r^{k}+\xi^{k}\right)+j_{-}^{i}\left(r^{k}-\xi^{k}\right) .
$$

In this case, there is no need for a counterpart of the last term in (87). For both, head-on and inspiral collisions, $j_{+}^{i}=-j_{-}^{i}=j_{*}^{i}$; thus,

$$
j^{i}\left(r^{k}\right)=j_{*}^{i}\left(r^{k}+\xi^{k}\right)-j_{*}^{i}\left(r^{k}-\xi^{k}\right),
$$

which obviously has the appropriate zero-separation limit, namely $j^{i}\left(r^{k}\right) \rightarrow 0$ as $\xi^{i} \rightarrow 0$. Once again, we apply the close-limit condition and approximate

$$
j_{*}^{i}\left(r^{k} \pm \xi^{k}\right)=j_{*}^{i}\left(r^{k}\right) \pm \xi^{j} \nabla_{j} j_{*}^{i}\left(r^{k}\right)+\frac{1}{2} \xi^{l} \xi^{m} \nabla_{l} \nabla_{m} j_{*}^{i}\left(r^{k}\right) .
$$

Therefore,

$$
j^{i}=2 \xi^{j} \nabla_{j} j_{*}^{i} .
$$

Notice that Eq. (94) directly gives the momentum density perturbation because, by construction, the background is static.

\section{A. Head-on Collision Initial Data}

For simplicity, we assume that the collision takes place along the $z$-axis. Therefore,

$$
\begin{aligned}
& \xi^{i}=\xi z^{i} \\
& j_{*}^{i}=-J_{*} z^{i},
\end{aligned}
$$


where

$$
z^{i}=\left(\cos \theta,-\frac{1}{r} \sin \theta, 0\right)
$$

is a unit vector along the $z$-axis.

Substitution of (95) into (90) yields

$$
\begin{aligned}
\sigma_{(1)} & =\sigma_{*}-\sigma_{(0)}+\frac{1}{2} \xi^{2}\left[\cos ^{2} \theta\left(\frac{d}{d r} \sigma_{*}\right)^{2}+\cos ^{2} \theta \frac{d^{2}}{d r^{2}} \sigma_{*}+\sin ^{2} \theta \frac{1}{r} \frac{d}{d r} \sigma_{*}\right] . \\
& =\sqrt{4 \pi} Y_{00}\left[\sigma_{*}-\sigma_{(0)}+\frac{1}{6} \xi^{2}\left\{\frac{d^{2}}{d r^{2}} \sigma_{*}+\left(\frac{d}{d r} \sigma_{*}\right)^{2}+\frac{2}{r} \frac{d}{d r} \sigma_{*}\right\}\right] \\
& +\frac{2}{15} \sqrt{5 \pi} Y_{20} \xi^{2}\left[\frac{d^{2}}{d r^{2}} \sigma_{*}+\left(\frac{d}{d r} \sigma_{*}\right)^{2}-\frac{1}{r} \frac{d}{d r} \sigma_{*}\right] .
\end{aligned}
$$

It is clear from (98) that the conformal density perturbation has a monopole contribution $(m=0, l=0)$. Since our main interest is associated with the gravitational waves generated during the merger, we will ignore this contribution; it obviously does not lead to any gravitational waves. The remaining part is the radiative quadrupole $(m=0, l=2)$ perturbation:

$$
\sigma_{(1)}=\frac{2}{15} \sqrt{5 \pi} \xi^{2}\left[\frac{d^{2}}{d r^{2}} \sigma_{*}+\left(\frac{d}{d r} \sigma_{*}\right)^{2}-\frac{1}{r} \frac{d}{d r} \sigma_{*}\right] .
$$

This initial data set is discussed in considerable detail in [6].

Let us now consider the momentum density perturbation. Substitution of (96) into (94) yields

$$
\begin{aligned}
j^{i} & =-2 \xi z^{j} \nabla_{j}\left(J_{*} z^{i}\right) \\
& =-2 \xi z^{i} z^{j} \nabla_{j} J_{*} \\
& =-2 \xi \frac{d}{d r} J_{*}\left(\cos ^{2} \theta,-\frac{1}{r} \sin \theta \cos \theta, 0\right) \\
& =-\frac{4}{3} \sqrt{\pi} \xi \frac{d}{d r} J_{*}\left(Y_{00}, 0,0\right)-\frac{8}{15} \sqrt{5 \pi} \xi \frac{d}{d r} J_{*}\left(Y_{20}, \frac{2}{r} \partial_{\theta} Y_{20}, 0\right) .
\end{aligned}
$$

As expected and in agreement with the density perturbation, we again have a monopole momentum density perturbation that we shall ignore. Comparing the quadrupole terms in (100) with (58), we have $J_{3}=0$ and

$$
J_{1}=2 J_{2}=-\frac{8}{15} \sqrt{5 \pi} \xi \frac{d}{d r} J_{*} .
$$

From Eq. (4) and (5) and the conformal transformations (15) and (16), the expressions for $\sigma_{*}$ and $J_{*}$ in Eqs. (99) and (101) are given by:

$$
\begin{aligned}
\sigma_{*} & =\hat{\rho}_{*} \phi_{(0)}^{8} \\
J_{*} & =\left(\hat{\rho}_{*}+\hat{p}_{*}\right) v \phi_{(0)}^{10},
\end{aligned}
$$

with $v$ the magnitude of the collision velocity. In writing the above expressions, we used that to zero-order $\hat{u}^{i}=0$ and $\hat{\gamma}=1+O\left(\hat{u}^{2}\right)$. Notice that the conformal factor in connection with the background star, $\phi_{(0)}$, was the one used in Eqs. (102) and (103) to transform the physical TOV solutions of the colliding stars since it is the background star that provides the conformal space where our calculations are performed. Also important is to notice that, once the background and colliding neutron stars models have been completely determined, there are only two parameters that characterize the initial data: the separation $\xi$ and the velocity $v$.

\section{B. Inspiral Collision Initial Data}

For this case, we assume that the initial configuration is such that the neutron stars are along the $x$-axis and their momentum pointing along the $y$-axis. Then 


$$
\begin{aligned}
& \xi^{i}=\xi x^{i} \\
& j_{*}^{i}=J_{*} y^{i},
\end{aligned}
$$

where

$$
\begin{aligned}
x^{i} & =\left(\sin \theta \cos \varphi, \frac{1}{r} \cos \theta \cos \varphi,-\frac{\sin \varphi}{r \sin \theta}\right) \\
y^{i} & =\left(\sin \theta \sin \varphi, \frac{1}{r} \cos \theta \sin \varphi, \frac{\cos \varphi}{r \sin \theta}\right) .
\end{aligned}
$$

are unit vectors along the $x$-axis and $y$-axis, respectively. Substitution of (104) and (105) into (90) yields

$$
\begin{aligned}
\sigma_{(1)} & =\sigma_{*}-\sigma_{(0)}+\frac{1}{2} \xi^{2}\left[\sin ^{2} \theta \cos ^{2} \varphi\left\{\left(\frac{d}{d r} \sigma_{*}\right)^{2}+\frac{d^{2}}{d r^{2}} \sigma_{*}\right\}\right. \\
& \left.+\left(\cos ^{2} \theta \cos ^{2} \varphi+\sin ^{2} \varphi\right) \frac{1}{r} \frac{d}{d r} \sigma_{*}\right] \\
& =\sqrt{4 \pi} Y_{00}\left[\sigma_{*}-\sigma_{(0)}+\frac{1}{6} \xi^{2}\left\{\frac{d^{2}}{d r^{2}} \sigma_{*}+\left(\frac{d}{d r} \sigma_{*}\right)^{2}+\frac{2}{r} \frac{d}{d r} \sigma_{*}\right\}\right] \\
& +\left(-\sqrt{\frac{\pi}{5}} Y_{20}+\sqrt{\frac{2 \pi}{15}} \operatorname{Re} Y_{22}\right) \xi^{2}\left[\frac{d^{2}}{d r^{2}} \sigma_{*}+\left(\frac{d}{d r} \sigma_{*}\right)^{2}-\frac{1}{r} \frac{d}{d r} \sigma_{*}\right] .
\end{aligned}
$$

As with the head-on collisions, we concentrate on the radiative quadrupole $(m=2, l=2)$ term, so the density perturbation $\sigma_{(1)}$ source of Eq. (61) is given by:

$$
\sigma_{(1)}=\sqrt{\frac{2 \pi}{15}} \xi^{2}\left[\frac{d^{2}}{d r^{2}} \sigma_{*}+\left(\frac{d}{d r} \sigma_{*}\right)^{2}-\frac{1}{r} \frac{d}{d r} \sigma_{*}\right] .
$$

Notice that the only difference between (109) and the corresponding source term in the head-on collision case, i.e. Eq. (99), is a numerical factor.

For the momentum density perturbation, substitution of (105) into (94) yields

$$
\begin{aligned}
j^{i} & =2 \xi x^{j} \nabla_{j}\left(J_{*} y^{i}\right) \\
& =2 \xi y^{i} x^{j} \nabla_{j} J_{*} \\
& =2 \xi \frac{d}{d r} J_{*}\left(\sin ^{2} \theta \sin \varphi \cos \varphi, \frac{1}{r} \sin \theta \cos \theta \sin \varphi \cos \varphi, \frac{1}{r} \cos ^{2} \varphi\right) \\
& =-\sqrt{\frac{4 \pi}{3}} \xi \frac{d}{d r} J_{*}\left(0,0, \frac{1}{r \sin \theta} \partial_{\theta} Y_{10}\right)+4 \sqrt{\frac{2 \pi}{15}} \xi \frac{d}{d r} J_{*} \operatorname{Im}\left(Y_{22}, \frac{1}{r} \partial_{\theta} Y_{22}, \frac{1}{r \sin ^{2} \theta} \partial_{\varphi} Y_{22}\right) .
\end{aligned}
$$

Comparing (110) with (58), we deduce for the quadrupole term $(m=2, l=2) J_{3}=0$ and

$$
J_{1}=2 J_{2}=4 \sqrt{\frac{2 \pi}{15}} \xi \frac{d}{d r} J_{*},
$$

The dipole term $(m=0, l=1)$ does not contribute to the emerging gravitational radiation and can be ignored. Once again, the momentum density perturbation for the inspiral case only differs from the head-on case by a numerical factor. The only non-trivial differences in the initial data will then arise from the $Y_{l m}$ 's since in one case $m=0$ (head-on) and for the other $m=2$ (inspiral). The quantities $\sigma_{*}$ and $J_{*}$ in (109) and (111) are obtained as in the head-on collision case, namely from Eqs. (102) and (103) respectively.

Figure 1 shows profiles of the density perturbation $\sigma_{(1)}$ and the momentum density perturbation $J_{1}$ for the closelimit, boosted, head-on collision. Recall that for inspiral and head-on collisions $J_{1}=2 J_{2}$ and $J_{3}=0$. The perturbations $\sigma_{(1)}$ and $J_{1}$ were calculated from neutron stars with initial separation $\xi=0.1 R_{(0)}$ and velocity $v=0.1 c$. The corresponding perturbations for the inspiral case only differ from the perturbations shown in Fig. I by a constant numerical factor (compare Eqs. (99) and (101) with Eqs. (109) and (111). The TOV parameters for the background and colliding stars are: $\rho_{c}^{(0)}=2.69 \times 10^{15} \mathrm{~g} / \mathrm{cm}^{3}$ and $\kappa_{(0)}=100 \mathrm{~km}^{2}$. For these parameters, the mass and radius of the 
background star are $M_{(0)}=1.24 M_{\odot}$ and $R_{(0)}=9.0 \mathrm{~km}$, respectively. The initial colliding stars, which are displaced a distance $0.1 R_{0}$ from the center of mass, follow from $\rho_{c}^{*}=2.98 \times 10^{15} \mathrm{~g} / \mathrm{cm}^{3}$ and $K_{*}=90.25 \mathrm{~km}^{2}$. With these parameters, the colliding stars have a mass and radius of $M_{*}=1.17 M_{\odot}$ and $R_{0} *=8.58 \mathrm{~km}$, respectively. Figure 2 shows the solutions to the conformal perturbation $\phi_{(1)}$ and the harmonic components $W_{1}$ and $W_{2}$ (see Eq. 60) of the vector $W^{i}$ for the close-limit collision of neutron stars corresponding to the perturbations in Fig.1.

\section{CONCLUDING REMARKS}

In this paper, we have presented a framework for constructing initial data relevant for perturbative studies of neutron stars. Our approach was to "linearize" Lichnerowicz-York's standard procedure for the initial-value problem in General Relativity, and it facilitates (to a certain extent) setting astrophysical initial data for perturbation evolutions, cf. [5]. It is straightforward to compare our method (as well as the results) to the fully nonlinear one, which is important since a main motivation for perturbation studies is to provide benchmark tests for nonlinear numerical relativity.

As examples of interesting initial data that can be constructed from our equations, we constructed data for merging neutron stars in the close-limit approximation. The simplest case of these data sets, that describe head-on collision of two initially static stars, has already been extensively discussed in [6]. No studies of the more general data with initial momentum and for inspiralling collisions have yet been performed. Such simulations should obviously be carried out, and we hope to be able to discuss the relevant results, as well as possible extensions of the framework developed in this paper to, for example, rotating configurations, in the near future.

\section{ACKNOWLEDGMENTS}

We thank Johannes Ruoff for his assistance with some of the tensor harmonics manipulations. This work was partially supported by NATO grants CRG960260 and CRG971092, as well as NSF grants PHY9800973 and PHY9357219.

[1] G.B. Cook, Phys. Rev. D, 44, 2983 (1991)

[2] J.W. York,in Sources of Gravitational Radiation, edited by L. Smarr (Cambridge University Press, Cambridge, England, 1979), p. 83.

[3] P. Anninos, R.H. Price, J. Pullin, E. Seidel, W.-M. Suen, Phys. Rev. D, 52, 4462 (1995)

[4] R.H. Price and J. Pullin, Phys. Rev. Lett. , 72, 3297 (1994)

[5] G. Allen, N. Andersson, K. Kokkotas and B. Schutz, Phys. Rev. D, 58, 124012 (1998)

[6] G. Allen, N. Andersson, K. Kokkotas, P. Laguna, J. Pullin and J. Ruoff, submitted to PRD (1999), gr-qc/9903100.

[7] R. Arnowitt, S. Deser and C.W. Misner, in Gravitation ed. L. Witten (Wiley, New York, 1962), p. 227.

[8] J.M. Bowen, Gen. Rel. Grav., 11, 227 (1979); Gen. Rel. Grav., 14, 1183 (1982)

[9] J.M. Bowen and J.W. York Phys. Rev. D, 21, 2047 (1983) 


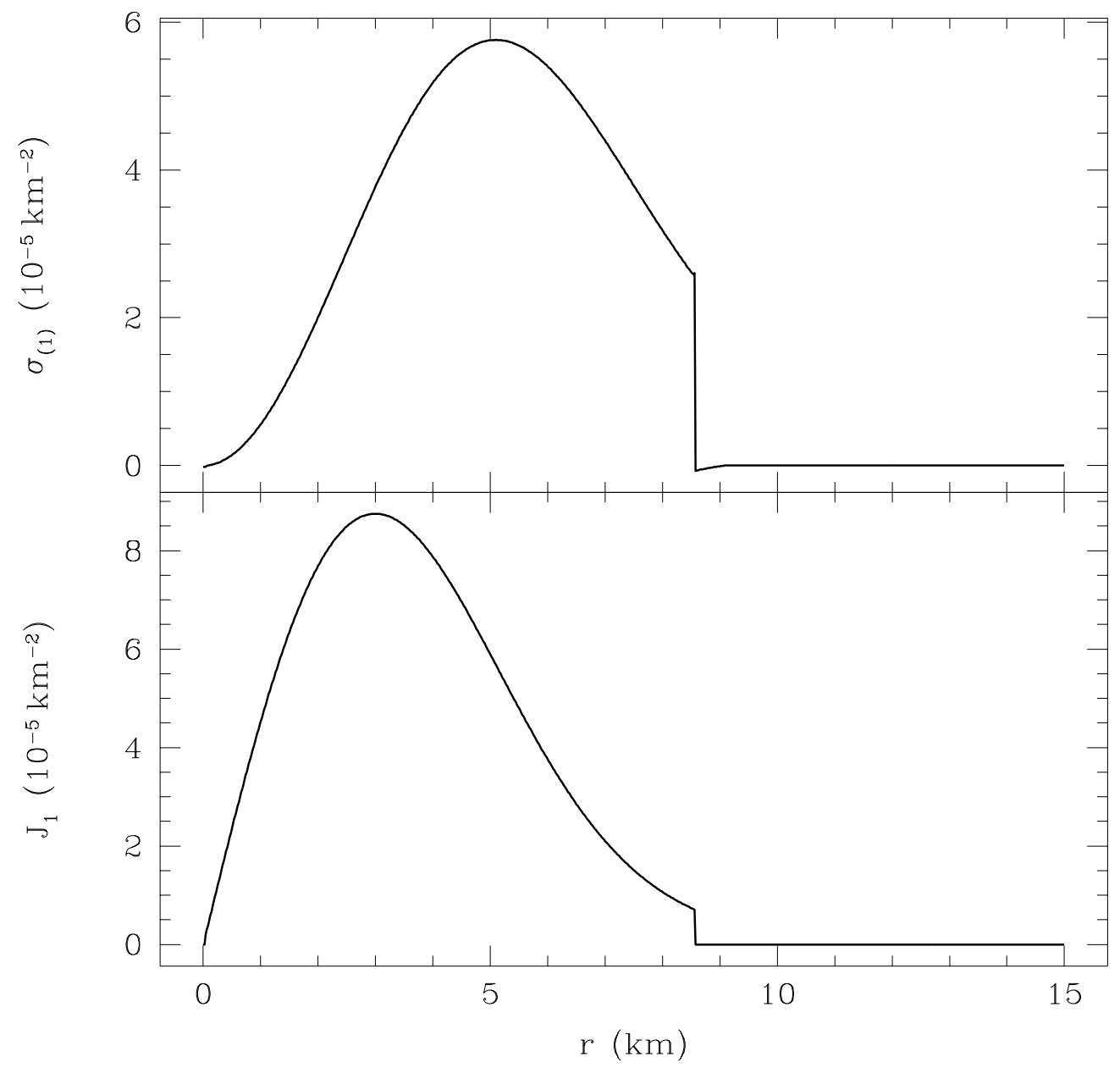

FIG. 1. Density $\sigma_{(1)}$ and momentum density $J_{1}$ perturbations (see Eqs. 99 and 101 in the text) for the close-limit, boosted, head-on collision of neutron stars with initial separation $\xi=0.1 R_{(0)}$ and velocity $v=0.1 c$. The corresponding perturbations for the inspiral case differ from these quantities by constant numerical factors. The TOV parameters for the background and colliding stars are: $\rho_{c}^{(0)}=2.69 \times 10^{15} \mathrm{~g} / \mathrm{cm}^{3}$ and $\kappa_{(0)}=100 \mathrm{~km}^{2}$. For these parameters, the mass and radius of the background star are $M_{(0)}=1.24 M_{\odot}$ and $R_{(0)}=9.0 \mathrm{~km}$, respectively. The initial colliding stars, which are displaced a distance $0.1 R_{0}$ from the center of mass, follow from $\rho_{c}^{*}=2.98 \times 10^{15} \mathrm{~g} / \mathrm{cm}^{3}$ and $K_{*}=90.25 \mathrm{~km}^{2}$. With these parameters, the colliding stars have a mass and radius of $M_{*}=1.17 M_{\odot}$ and $R_{0} *=8.58 \mathrm{~km}$, respectively. 


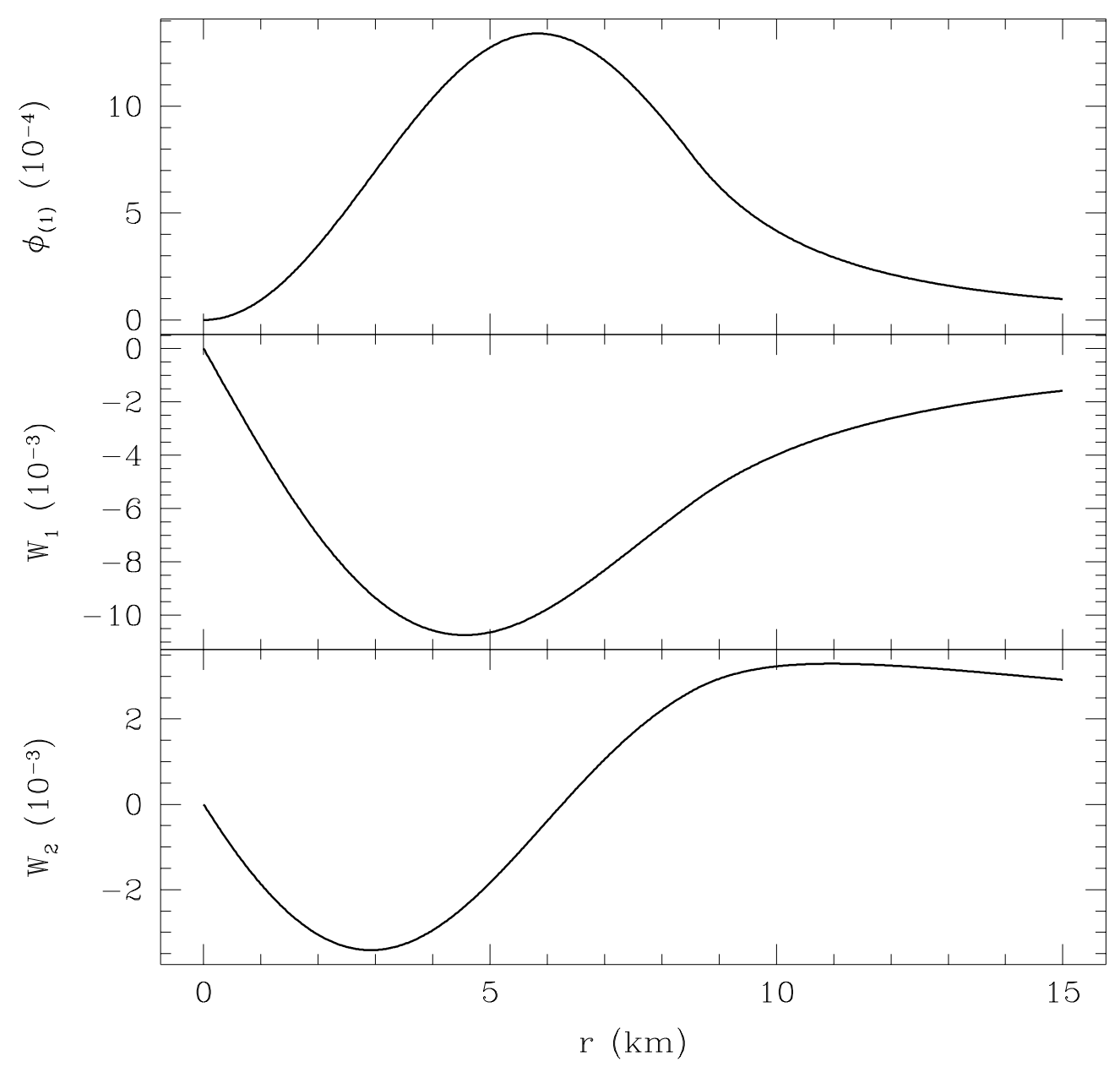

FIG. 2. Conformal perturbation $\phi_{(1)}$ and harmonic components $W_{1}$ and $W_{2}$ of the vector $W^{i}$ from the solution to the linearized constraints for the close-limit collision of neutron stars corresponding to the perturbations in Fig. 1 . 